\title{
A useful result related with zeros of continuous compactly supported mother wavelets
}

\author{
Antoine Ayache* and Céline Esser ${ }^{\dagger}$ \\ Laboratoire Paul Painlevé, Université Lille 1 \\ 59655 Villeneuve d'Ascq, France \\ *Antoine.Ayache@math.univ-lille1.fr \\ ${ }^{\dagger}$ Celine.Esser@math.univ-lille1.fr
}

Received 25 March 2016

Revised 7 February 2017

Accepted 15 May 2017

Published 9 June 2017

\begin{abstract}
In the last two decades, wavelet bases and associated methodologies have become quite important in many domains, such as signal and image processing, harmonic analysis, statistics, and so on. More recently, they also turn out to be quite useful in the probabilistic framework of stochastic processes, in which, among other things, they allow to obtain fine results concerning erratic sample paths behavior. The goal of our paper is to derive a result, related with zeros of continuous compactly supported mother wavelets, which is useful in this probabilistic framework. More precisely, let $\psi$ be any arbitrary such wavelet; we show that being given an arbitrary point $x_{0} \in \mathbb{R}$ there always exists at least one integer $k_{x_{0}} \in \mathbb{Z}$ such that $\psi\left(x_{0}-k_{x_{0}}\right) \neq 0$.
\end{abstract}

Keywords: Fourier methods; multiresolution analysis; two-scale equations; conjugate quadrature filter; trigonometric polynomials; wavelets.

AMS Subject Classification: 42C40, 42A05

\section{Introduction}

Throughout our paper we define a mother wavelet to be a function in the Hilbert space $L^{2}(\mathbb{R})$, denoted by $\psi$, with the property that the following collection of dilated and translated copies of $\psi$ :

$$
\left\{2^{\frac{j}{2}} \psi\left(2^{j} \cdot-k\right): j \in \mathbb{Z} \text { and } k \in \mathbb{Z}\right\}
$$

forms a tight frame of $L^{2}(\mathbb{R})$. The notion of tight frame is defined in e.g. Sec. 3.2 of Ref. 11. We point out that it is a more general notion than the usual one of orthonormal basis. Thus, the framework of our paper includes the classical one of compactly supported wavelet bases stemming from multiresolution analyses.

A real-valued compactly supported mother wavelet can be constructed starting from a two-scale functional equation (also called a refinement equation), in the space 
$L^{2}(\mathbb{R})$, of the form:

$$
\varphi(x)=2 \sum_{l=0}^{2 N-1} a_{l} \varphi(2 x-l), \quad \forall x \in \mathbb{R},
$$

where the integer $N \geq 1$ and the real-valued coefficients $a_{0}, \ldots, a_{2 N-1}$ are fixed and such that $a_{0} \cdot a_{2 N-1} \neq 0$. This kind of equations was first introduced by de Rham in 1956 in order to construct continuous but nowhere differentiable functions 12 Later, refinement equations were reintroduced in the context of interpolation algorithms. $13 \sqrt[24]{ }$ However, in contrast with de Rham's work, the aim was to construct curves as regular as possible. In particular, these interpolation algorithms have applications in computer-aided design (CAD) since they allow to smooth polyhedral curves.

Let us now recall precisely some important connections between a mother wavelet and a two-scale functional equation of the type (II). First, we mention that, throughout our paper, we use the convention that the Fourier transform of an arbitrary function $f \in L^{1}(\mathbb{R})$ is defined, for each $\xi \in \mathbb{R}$, as $\widehat{f}(\xi):=\int_{\mathbb{R}} e^{-i \xi x} f(x) d x$. Also, we mention that the Fourier transform of an arbitrary function $g$ in $L^{2}(\mathbb{R})$ is defined as $\widehat{g}=\lim _{n \rightarrow+\infty} \widehat{f}_{n}$, where the convergence holds in $L^{2}(\mathbb{R})$, and where $\left(f_{n}\right)_{n}$ is a sequence of functions belonging to $L^{1}(\mathbb{R}) \cap L^{2}(\mathbb{R})$ and converging to $g$ in $L^{2}(\mathbb{R})$. Then, notice that the functional equation (1) can be rewritten in the Fourier domain as

$$
\widehat{\varphi}(2 \xi)=m_{0}(\xi) \widehat{\varphi}(\xi), \quad \forall \xi \in \mathbb{R},
$$

where the trigonometric polynomial

$$
m_{0}(\xi):=\sum_{l=0}^{2 N-1} a_{l} e^{-i l \xi}, \quad \forall \xi \in \mathbb{R},
$$

is called the low-pass filter. Also, recall that the trigonometric polynomial $m_{1}$ defined as

$$
m_{1}(\xi):=\overline{m_{0}(\xi+\pi)} e^{-i \xi}, \quad \forall \xi \in \mathbb{R},
$$

is called the high-pass filter. We always impose to $m_{0}$ to satisfy the usual condition:

$$
\left\{\begin{array}{l}
m_{0}(0)=1, \\
\left|m_{0}(\xi)\right|^{2}+\left|m_{0}(\xi+\pi)\right|^{2}=1, \quad \forall \xi \in \mathbb{R} .
\end{array}\right.
$$

Thanks to (4), the two-scale equation (1) has a unique solution, denoted by $\varphi$, which belongs to the space $L^{2}(\mathbb{R})$ (see e.g. Ref. 18 and Sec. 6.2 of Ref. 11). The function $\varphi$ is called a refinable function. Observe that its Fourier transform satisfies

$$
\widehat{\varphi}(\xi)=\prod_{j=1}^{+\infty} m_{0}\left(2^{-j} \xi\right), \quad \forall \xi \in \mathbb{R},
$$

where the infinite product converges pointwise and uniformly on each compact subset of $\mathbb{R}$ (see e.g. Sec. 6.2 of Ref. 11 and Sec. 2.3 of Ref. 15). Also observe that 
the function $\varphi$ is compactly supported, $\frac{18 \sqrt{20}}{m o r e}$ precisely, one has

$$
\operatorname{supp}(\varphi) \subseteq[0,2 N-1] .
$$

Now, let $m_{1}$ be the same trigonometric polynomial as in (3). One of the most fundamental results of the wavelet theory is that the function $\psi$ defined in the Fourier domain through the relation

$$
\widehat{\psi}(2 \xi):=m_{1}(\xi) \widehat{\varphi}(\xi), \quad \forall \xi \in \mathbb{R},
$$

is a mother wavelet (see e.g. Ref. 18 and Proposition 6.2.3 of Ref. 11). Notice that it can easily be derived from (6) and (5) that $\psi$ is a compactly supported function satisfying $\operatorname{supp}(\psi) \subseteq[-N+1, N]$. Also, we mention that a simple sufficient, but not necessary, condition for the tight frame

$$
\left\{2^{\frac{j}{2}} \psi\left(2^{j} \cdot-k\right): j \in \mathbb{Z} \text { and } k \in \mathbb{Z}\right\}
$$

to be an orthonormal basis of $L^{2}(\mathbb{R})$ is that the low-pass filter $m_{0}$ vanishes nowhere on the interval $[-\pi / 3, \pi / 3]$ (see for instance Corollary 6.3.2 of Ref. 11).

Let us now explain the motivation behind our paper. In the last two decades, wavelet bases and associated methodologies have become quite important in many domains, such as signal and image processing, harmonic analysis, statistics, and so on. More recently, they also turn out to be quite useful in the probabilistic framework of stochastic processes, in which, among other things, they allow to obtain fine results concerning erratic sample paths behavior (see e.g. Refs. 8 [23, 3] 7, 25, 4, 5, 16, 1 and 17). In this probabilistic framework, it is useful that the mother wavelet $\psi$ be a continuous (or more regular) function satisfying the following property:

$(\mathcal{P})$ the 1-periodic continuous functions of the family $x \in \mathbb{R} \mapsto \sum_{k \in \mathbb{Z}}|\psi(x-k)|^{\alpha}$, with $\alpha$ an arbitrary positive fixed parameter, never vanish.

Indeed, this property $(\mathcal{P})$, or some variants of it, plays a crucial role at least in the three papers $326 \mid 6$ as well as in the Ph.D. thesis $\frac{25}{25}$ The goal of our paper is to show that any arbitrary continuous compactly supported mother wavelet $\psi$ satisfies $(\mathcal{P})$. Before ending this introduction, we mention that $(\mathcal{P})$ can equivalently be expressed in the following two ways:

(i) being given an arbitrary point $x_{0} \in \mathbb{R}$ there always exists at least one integer $k_{x_{0}} \in \mathbb{Z}$ such that $\psi\left(x_{0}-k_{x_{0}}\right) \neq 0$

(ii) one has $\bigcap_{k \in \mathbb{Z}}(Z+k)=\emptyset$, where $\emptyset$ denotes the empty set, $Z:=\{x \in \mathbb{R}: \psi(x)=$ $0\}$ is the set of the zeros of $\psi$, and $(Z+k):=\{x+k: x \in Z\}$, for any fixed $k \in \mathbb{Z}$.

\section{Statement of the Main Result and Further Remarks}

Theorem 2.1. Let $\varphi$ be the unique solution of a two-scale functional equation of the form (1), and let $\psi$ be the compactly supported mother wavelet defined in terms of $\varphi$ through (6). If $\varphi$ and $\psi$ are continuous functions over the real line, then being 
given an arbitrary point $x_{0} \in \mathbb{R}$ there always exists at least one integer $k_{x_{0}} \in \mathbb{Z}$ such that $\psi\left(x_{0}-k_{x_{0}}\right) \neq 0$.

The proof of Theorem 2.1 is postponed to Sec. 3 The remaining of the present section is devoted to some remarks related with this theorem.

Remark 2.2. Theorem 2.1 becomes an obvious result when one replaces in its statement $\psi\left(x_{0}-k_{x_{0}}\right) \neq 0$ by $\varphi\left(x_{0}-k_{x_{0}}\right) \neq 0$. Indeed, under the condition (4) on the low-pass filter $m_{0}$, it has been shown (see Ref. 18 for instance) that the integer translates of $\varphi$ form a partition of unity, that is, for every $x \in \mathbb{R}$, one has

$$
\sum_{k \in \mathbb{Z}} \varphi(x-k)=1
$$

Remark 2.3. In the particular case of a non-compactly supported Lemarié-Meyer mother wavelet (see e.g. Refs. 21, 22 and 11), denoted by $\Psi$ (to be distinguished from a compactly supported mother wavelet $\psi$ ), results similar to Theorem 2.1 have already been obtained in Refs. 3, 6, 25] and 26. Since they are based on the specific properties (a) and (b) of $\Psi$ given below, the proofs of these earlier results are different from the one which allows us in the next section to derive Theorem 2.1]

For the sake of completeness, let us now briefly present the main lines of these proofs in Refs. 3, 6] 25] and 26, First, we recall that $\Psi$ satisfies the following two important "nice" properties:

(a) $\Psi$ belongs to the Schwartz class $\mathcal{S}(\mathbb{R})$, that is the space of the infinitely differentiable functions over the real line which, as well as all their derivatives of any order, vanish at infinity faster than any polynomial;

(b) the Fourier transform of $\Psi$ is a compactly supported function satisfying

$$
\operatorname{supp} \widehat{\Psi} \subseteq\left\{\xi \in \mathbb{R}: \frac{2 \pi}{3} \leq|\xi| \leq \frac{8 \pi}{3}\right\} .
$$

The strategy employed in Refs. [3, 6, 25] and 26] consists in arguing by contradiction. So, one starts from the assumption that the equality $\Psi\left(x_{0}-k\right)=0$ holds, for some $x_{0} \in \mathbb{R}$ and for every $k \in \mathbb{Z}$. Then, it turns out that all the Fourier coefficients of the $2 \pi$-periodic continuous function

$$
\xi \mapsto F_{x_{0}}(\xi):=\sum_{m \in \mathbb{Z}} e^{i x_{0}(\xi+2 m \pi)} \widehat{\Psi}(\xi+2 \pi m)
$$

vanish. Thus, for all $\xi \in \mathbb{R}$, one has $F_{x_{0}}(\xi)=0$, which in particular implies that $F_{x_{0}}(4 \pi / 3)=0$. The latter equality combined with (8) and the property (b) implies that $\widehat{\Psi}(4 \pi / 3)=0$. This is impossible since the same property (b) and the classical equality (see e.g. Refs. 22 and 11)

$$
\sum_{m \in \mathbb{Z}}|\widehat{\Psi}(\xi+2 \pi m)|^{2}=1, \quad \forall \xi \in \mathbb{R},
$$

entail that $\widehat{\Psi}(4 \pi / 3) \neq 0$. 
Remark 2.4. It is worth mentioning that a property for mother wavelets, very similar to our Theorem 2.1, is needed in Ref. 14 for determining rates of divergence of some wavelet series. More precisely, it is proved that if the corresponding mother wavelet, denoted by $\check{\psi}$, is a continuous function on the real line whose support can be unbounded (this is the sole difference with $\psi$ in Theorem [2.1), it satisfies the following property: there exists a finite set $\mathcal{I}$ included in $\mathbb{N} \times \mathbb{Z}$ such that

$$
\inf _{x \in[0,1]}\left\{\sum_{(j, k) \in \mathcal{I}}\left|\check{\psi}\left(2^{j} x-k\right)\right|\right\}>0 .
$$

Remark 2.5. Last but not least, we mention that in Ref. 2, in which the multifractal behavior of traces of Besov functions is investigated, it is needed that the smooth mother wavelet $\psi$, used in this study, satisfies a property bearing some resemblance to our Theorem 2.1 More precisely, letting $G_{\psi}$ be the periodization of $\psi$ defined, for all $x \in \mathbb{R}$, as

$$
G_{\psi}(x):=\sum_{k \in \mathbb{Z}} \psi(x-k),
$$

it is needed that the restriction of $G_{\psi}$ to the interval $[0,1]$ vanishes only on a finite number of points, and also that the derivative $G_{\psi}^{\prime}$ vanishes at no one of these points. It has been shown in Ref. 2 that a Lemarié-Meyer mother wavelet $\Psi$ satisfies such a property; it would be interesting and useful to show that other classes of mother wavelets $\psi$ share the same property. We mention that in the case of a Lemarié-Meyer mother wavelet $\Psi$, the proof of this fact has been done in Ref. 2 in a very simple way. Namely, the use of Poisson's formula and the property (b) of $\Psi$ gives

$$
G_{\Psi}(x)=\sum_{k \in \mathbb{Z}} \widehat{\Psi}(2 \pi k) e^{2 i \pi k x}=\widehat{\Psi}(-2 \pi) e^{-2 i \pi x}+\widehat{\Psi}(2 \pi) e^{2 i \pi x}=-\sqrt{2} \cos (2 \pi x),
$$

where the last equality results from the fact that $\widehat{\Psi}(-2 \pi)=\widehat{\Psi}(2 \pi)=-\sqrt{2} / 2$ (see Sec. 5.2 and relations (4.2.4) and (4.2.5) in Ref. 11). Thus, it turns out that the set $\left\{x \in[0,1]: G_{\Psi}(x)=0\right\}$ reduces to $\{1 / 4,3 / 4\}$ and that one has $G_{\Psi}^{\prime}(1 / 4) \neq 0$ and $G_{\Psi}^{\prime}(3 / 4) \neq 0$.

\section{Proof of the Main Result}

First we need to obtain some preliminary results and to introduce some additional notations.

Definition 3.1. Let $\left(c_{k}\right)_{k \in \mathbb{Z}}$ be an arbitrary sequence of complex numbers having a finite support, that is the set $\left\{k \in \mathbb{Z}: c_{k} \neq 0\right\}$ is finite. Let $\lambda$ be the trigonometric polynomial defined, for all $\xi \in \mathbb{R}$, as

$$
\lambda(\xi)=\sum_{k \in \mathbb{Z}} c_{k} e^{i k \xi} .
$$


Then the even and odd parts of $\lambda$ are the trigonometric polynomials, respectively denoted by $\lambda_{e}$ and $\lambda_{o}$, defined, for all $\xi \in \mathbb{R}$, as

$$
\lambda_{e}(\xi):=2^{-1}(\lambda(\xi)+\lambda(\xi+\pi))=\sum_{k \in \mathbb{Z}} c_{2 k} e^{2 i k \xi}
$$

and

$$
\lambda_{o}(\xi):=2^{-1}(\lambda(\xi)-\lambda(\xi+\pi))=\sum_{k \in \mathbb{Z}} c_{2 k+1} e^{i(2 k+1) \xi} .
$$

Lemma 3.2. Let $\left(a_{l}\right)_{0 \leq l \leq 2 N-1}$ be the sequence of the real-valued coefficients of the low-pass filter $m_{0}$ which is defined in (2) and which satisfies (4). Then the polynomial functions of the complex variable $z$

$$
P_{e}(z):=\sum_{l=0}^{N-1} a_{2 l} z^{2 l} \quad \text { and } \quad P_{o}(z):=\sum_{l=0}^{N-1} a_{2 l+1} z^{2 l+1}
$$

are coprime.

Proof. Let us consider the even and odd parts of the trigonometric polynomial $m_{0}$, that is (see Definition [3.1) the trigonometric polynomials $m_{0, e}$ and $m_{0, o}$ defined, for all $\xi \in \mathbb{R}$, as

$$
m_{0, e}(\xi):=\sum_{l=0}^{N-1} a_{2 l} e^{-2 i l \xi} \text { and } m_{0, o}(\xi):=\sum_{l=0}^{N-1} a_{2 l+1} e^{-i(2 l+1) \xi} .
$$

In view of the fact that $m_{0, e}(\xi+\pi)=m_{0, e}(\xi)$ and $m_{0, o}(\xi+\pi)=-m_{0, o}(\xi)$, it easily follows from (4) that

$$
\left|m_{0, e}(\xi)\right|^{2}+\left|m_{0, o}(\xi)\right|^{2}=\frac{1}{2}, \quad \forall \xi \in \mathbb{R} .
$$

Thus, using (10) we get, for every $\xi \in \mathbb{R}$, that

$$
\begin{aligned}
& \left(\sum_{l=0}^{N-1} a_{2 l} e^{-2 i l \xi}\right)\left(\sum_{l=0}^{N-1} a_{2 l} e^{2 i l \xi}\right) \\
& \quad+\left(\sum_{l=0}^{N-1} a_{2 l+1} e^{-i(2 l+1) \xi}\right)\left(\sum_{l=0}^{N-1} a_{2 l+1} e^{i(2 l+1) \xi}\right)=\frac{1}{2} .
\end{aligned}
$$

Next multiplying both sides of this equality by $e^{i(2 N-1) \xi}$, we obtain that

$$
\begin{aligned}
& \left(\sum_{l=0}^{N-1} a_{2 l} e^{i(2 N-1-2 l) \xi}\right)\left(\sum_{l=0}^{N-1} a_{2 l} e^{2 i l \xi}\right) \\
& \quad+\left(\sum_{l=0}^{N-1} a_{2 l+1} e^{i(2 N-2 l-2) \xi}\right)\left(\sum_{l=0}^{N-1} a_{2 l+1} e^{i(2 l+1) \xi}\right)=\frac{e^{i(2 N-1) \xi}}{2}
\end{aligned}
$$


which, in view of (9), implies that, for every $z \in \mathbb{C}$, one has

$$
\left(\sum_{l=0}^{N-1} a_{2 l} z^{2 N-1-2 l}\right) P_{e}(z)+\left(\sum_{l=0}^{N-1} a_{2 l+1} z^{2 N-2 l-2}\right) P_{o}(z)=\frac{z^{2 N-1}}{2} .
$$

Consequently, if the polynomials $P_{e}$ and $P_{o}$ have a common root, this root can only be 0 . But $P_{e}(0)=a_{0} \neq 0$ by assumption and therefore, $P_{e}$ and $P_{o}$ are coprime.

The following remark is a classical result; it has a very short proof which is given for the sake of completeness.

Remark 3.3. One has $\sum_{l=0}^{N-1} a_{2 l}=\sum_{l=0}^{N-1} a_{2 l+1}=1 / 2$.

Proof. As done previously, we consider the polynomials $m_{0, e}$ and $m_{0, o}$ defined by (10). Evaluating $m_{0}$ at 0 and $\pi$ and using (4), we get that

$$
\left\{\begin{array}{l}
1=m_{0}(0)=m_{0, e}(0)+m_{0, o}(0)=\sum_{l=0}^{N-1} a_{2 l}+\sum_{l=0}^{N-1} a_{2 l+1}, \\
0=m_{0}(\pi)=m_{0, e}(\pi)+m_{0, o}(\pi)=\sum_{l=0}^{N-1} a_{2 l}-\sum_{p=0}^{N-1} a_{2 l+1},
\end{array}\right.
$$

which implies that $\sum_{l=0}^{N-1} a_{2 l}=\sum_{l=0}^{N-1} a_{2 l+1}=1 / 2$.

For the sake of convenience, we set $a_{l}:=0$ for all $l \in \mathbb{Z} \backslash\{0, \ldots, 2 N-1\}$. Then the high-pass filter $m_{1}$, defined in (3), can be expressed as

$$
m_{1}(\xi)=\sum_{l \in \mathbb{Z}} b_{l} e^{-i l \xi}, \quad \forall \xi \in \mathbb{R},
$$

where

$$
b_{l}:=(-1)^{l-1} a_{1-l}, \quad \forall l \in \mathbb{Z} .
$$

Remark that the mother wavelet defined via (6) satisfies in the time domain

$$
\psi(x)=2 \sum_{l \in \mathbb{Z}} b_{l} \varphi(2 x-l), \quad \forall x \in \mathbb{R} .
$$

Note that this last relation holds at every point $x \in \mathbb{R}$ thanks to the assumption of continuity of the functions $\varphi$ and $\psi$.

We are now ready to prove Theorem 2.1.

Proof of Theorem 2.1. Given an arbitrary fixed $x_{0} \in \mathbb{R}$, let us consider the trigonometric polynomial $\mu_{x_{0}}$ defined, for all $\xi \in \mathbb{R}$, as

$$
\mu_{x_{0}}(\xi):=\sum_{m \in \mathbb{Z}} \psi\left(x_{0}-m\right) e^{-2 i m \xi} ;
$$

this is in fact a finite sum since the mother wavelet $\psi$ is compactly supported. Notice that, in order to obtain the theorem, it is enough to show that $\mu_{x_{0}}$ does 
not vanish everywhere. Using (13), one can express $\mu_{x_{0}}$ in terms of the refinable function $\varphi$ in the following way:

$$
\mu_{x_{0}}(\xi)=2 \sum_{m \in \mathbb{Z}} \sum_{l \in \mathbb{Z}} b_{l} \varphi\left(2 x_{0}-2 m-l\right) e^{-2 i m \xi}, \quad \forall \xi \in \mathbb{R} .
$$

On the other hand, let $\nu_{x_{0}}$ be the trigonometric polynomial defined, for all $\xi \in \mathbb{R}$, as

$$
\nu_{x_{0}}(\xi):=\sum_{m \in \mathbb{Z}} \varphi\left(2 x_{0}-m\right) e^{-i m \xi} ;
$$

this is again a finite sum since the refinable function $\varphi$ is compactly supported. Notice that one can derive from (11) that

$$
\begin{aligned}
\overline{m_{1}(\xi)} \nu_{x_{0}}(\xi) & =\sum_{l \in \mathbb{Z}} b_{l} e^{i l \xi} \sum_{m \in \mathbb{Z}} \varphi\left(2 x_{0}-m\right) e^{-i m \xi} \\
& =\sum_{l \in \mathbb{Z}} \sum_{m \in \mathbb{Z}} b_{l} \varphi\left(2 x_{0}-m\right) e^{i(l-m) \xi} \\
& =\sum_{l \in \mathbb{Z}} \sum_{p \in \mathbb{Z}} b_{l} \varphi\left(2 x_{0}-l-p\right) e^{-i p \xi}
\end{aligned}
$$

for each $\xi \in \mathbb{R}$. Combining these equalities with (14), it follows that the trigonometric polynomial $\mu_{x_{0}}(\xi)$ is the even part (see Definition 3.1) of the trigonometric polynomial $2 \overline{m_{1}(\xi)} \nu_{x_{0}}(\xi)$. Therefore, we have that

$$
\mu_{x_{0}}(\xi)=2\left(\overline{m_{1, e}(\xi)} \nu_{x_{0}, e}(\xi)+\overline{m_{1, o}(\xi)} \nu_{x_{0}, o}(\xi)\right), \quad \forall \xi \in \mathbb{R} .
$$

Let us proceed by contradiction and assume that, for every $\xi \in \mathbb{R}$, we have $\mu_{x_{0}}(\xi)=0$. Then the equality (15) reduces to

$$
\overline{m_{1, e}(\xi)} \nu_{x_{0}, e}(\xi)=-\overline{m_{1, o}(\xi)} \nu_{x_{0}, o}(\xi), \quad \forall \xi \in \mathbb{R} .
$$

That is, for all $\xi \in \mathbb{R}$, we have

$$
\begin{aligned}
& \left(\sum_{l \in \mathbb{Z}} b_{2 l} e^{2 i l \xi}\right)\left(\sum_{q \in \mathbb{Z}} \varphi\left(2 x_{0}-2 q\right) e^{-2 i q \xi}\right) \\
& =-\left(\sum_{l \in \mathbb{Z}} b_{2 l+1} e^{i(2 l+1) \xi}\right)\left(\sum_{q \in \mathbb{Z}} \varphi\left(2 x_{0}-2 q-1\right) e^{-i(2 q+1) \xi}\right) .
\end{aligned}
$$

In view of (12) and (5), this amounts to saying that, for each $\xi \in \mathbb{R}$,

$$
\begin{aligned}
& \left(\sum_{l=0}^{N-1} a_{2 l+1} e^{-2 i l \xi}\right)\left(\sum_{\left\lfloor 2 x_{0}-2 N+1\right\rfloor \leq 2 q \leq\left\lfloor 2 x_{0}\right\rfloor} \varphi\left(2 x_{0}-2 q\right) e^{-2 i q \xi}\right) \\
& \quad=\left(\sum_{l=0}^{N-1} a_{2 l} e^{-i(2 l-1) \xi}\right)\left(\sum_{\left\lfloor 2 x_{0}-2 N+1\right\rfloor \leq 2 q+1 \leq\left\lfloor 2 x_{0}\right\rfloor} \varphi\left(2 x_{0}-2 q-1\right) e^{-i(2 q+1) \xi}\right),
\end{aligned}
$$


where $\lfloor\cdot\rfloor$ denotes the integer part function. Multiplying both sides of the last equality by $e^{-i \xi} e^{i\left\lfloor 2 x_{0}-2 N+1\right\rfloor \xi}$, we obtain that

$$
\begin{aligned}
& \left(\sum_{l=0}^{N-1} a_{2 l+1} e^{-i(2 l+1) \xi}\right)\left(\sum_{\left\lfloor 2 x_{0}-2 N+1\right\rfloor \leq 2 q \leq\left\lfloor 2 x_{0}\right\rfloor} \varphi\left(2 x_{0}-2 q\right) e^{-i\left(2 q-\left\lfloor 2 x_{0}-2 N+1\right\rfloor\right) \xi}\right) \\
& =\left(\sum_{l=0}^{N-1} a_{2 l} e^{-2 i l \xi}\right) \\
& \quad \times\left(\sum_{\left\lfloor 2 x_{0}-2 N+1\right\rfloor \leq 2 q+1 \leq\left\lfloor 2 x_{0}\right\rfloor} \varphi\left(2 x_{0}-2 q-1\right) e^{-i\left(2 q+1-\left\lfloor 2 x_{0}-2 N+1\right\rfloor\right) \xi}\right),
\end{aligned}
$$

for every $\xi \in \mathbb{R}$. This equality of trigonometric polynomials gives an equality between the corresponding polynomials. More precisely, for each $z \in \mathbb{C}$, we have

$$
\begin{aligned}
& \left(\sum_{l=0}^{N-1} a_{2 l+1} z^{2 l+1}\right)\left(\sum_{\left\lfloor 2 x_{0}-2 N+1\right\rfloor \leq 2 q \leq\left\lfloor 2 x_{0}\right\rfloor} \varphi\left(2 x_{0}-2 q\right) z^{2 q-\left\lfloor 2 x_{0}-2 N+1\right\rfloor}\right) \\
& \quad=\left(\sum_{l=0}^{N-1} a_{2 l} z^{2 l}\right)\left(\sum_{\left\lfloor 2 x_{0}-2 N+1\right\rfloor \leq 2 q+1 \leq\left\lfloor 2 x_{0}\right\rfloor} \varphi\left(2 x_{0}-2 q-1\right) z^{2 q+1-\left\lfloor 2 x_{0}-2 N+1\right\rfloor}\right) .
\end{aligned}
$$

Let us now consider the polynomials $Q_{1}$ and $Q_{2}$ defined by

$$
\left\{\begin{array}{l}
Q_{1}(z):=\sum_{\left\lfloor 2 x_{0}-2 N+1\right\rfloor \leq 2 q \leq\left\lfloor 2 x_{0}\right\rfloor} \varphi\left(2 x_{0}-2 q\right) z^{2 q-\left\lfloor 2 x_{0}-2 N+1\right\rfloor,} \\
Q_{2}(z):=\sum_{\left\lfloor 2 x_{0}-2 N+1\right\rfloor \leq 2 q+1 \leq\left\lfloor 2 x_{0}\right\rfloor} \varphi\left(2 x_{0}-2 q-1\right) z^{2 q+1-\left\lfloor 2 x_{0}-2 N+1\right\rfloor .}
\end{array}\right.
$$

Evaluating (16) at $z=1$ and using Remark 3.3, we get

$$
\sum_{\left\lfloor 2 x_{0}-2 N+1\right\rfloor \leq 2 q \leq\left\lfloor 2 x_{0}\right\rfloor} \varphi\left(2 x_{0}-2 q\right)=\sum_{\left\lfloor 2 x_{0}-2 N+1\right\rfloor \leq 2 q+1 \leq\left\lfloor 2 x_{0}\right\rfloor} \varphi\left(2 x_{0}-2 q-1\right)
$$

i.e. $Q_{1}(1)=Q_{2}(1)$, and the equality

$$
\sum_{m \in \mathbb{Z}} \varphi\left(2 x_{0}-m\right)=1
$$

given in (7) leads to

$$
Q_{1}(1)=Q_{2}(1)=\frac{1}{2}
$$

Next, we recall that we know from Lemma 3.2, that the polynomials

$$
P_{o}(z):=\sum_{l=0}^{N-1} a_{2 l+1} z^{2 l+1} \quad \text { and } \quad P_{e}(z):=\sum_{l=0}^{N-1} a_{2 l} z^{2 l}
$$


are coprime. Thus, we can derive from (16) that $P_{e}$ divides $Q_{1}$, and $P_{o}$ divides $Q_{2}$. Remark that $\operatorname{deg}\left(Q_{2}\right) \leq\left\lfloor 2 x_{0}\right\rfloor-\left\lfloor 2 x_{0}-2 N+1\right\rfloor<2 N$. Moreover, $\operatorname{deg}\left(P_{o}\right)=2 N-1$ since $a_{2 N-1} \neq 0$ by assumption. It follows that $\operatorname{deg}\left(Q_{2}\right)=2 N-1$; thus, there exists $\zeta \in \mathbb{C}$ such that $Q_{2}=\zeta P_{o}$. Moreover, Remark 3.3 gives $P_{o}(1)=\frac{1}{2}$ and consequently, we obtain from (17) that $\zeta=1$ i.e.

$$
Q_{2}=P_{o}
$$

In particular, $Q_{2}$ is an odd polynomial, thus $\left\lfloor 2 x_{0}-2 N+1\right\rfloor$ has to be even. Hence, one gets that

$$
Q_{1}(0)=\varphi\left(2 x_{0}-\left\lfloor 2 x_{0}-2 N+1\right\rfloor\right)=0,
$$

where the last equality results from (5), since $2 x_{0}-2 N+1-\left\lfloor 2 x_{0}-2 N+1\right\rfloor \geq 0$. Note that (16) and (18) also lead to $Q_{1}=P_{e}$, which contradicts (19) since $P_{e}(0)=a_{0} \neq 0$. This concludes the proof of Theorem 2.1.

Remark 3.4 (Biorthogonal case). Let us end this note by a short comment concerning biorthogonal wavelets. For more information about this framework, we refer the reader to Refs. 10, 19 and Chap. 2 of Ref. 9] We consider a dual pair $(\varphi, \widetilde{\varphi})$ of continuous compactly supported refinable functions satisfying

$$
\varphi(x)=2 \sum_{l=N_{1}}^{N_{2}} a_{l} \varphi(2 x-l) \text { and } \widetilde{\varphi}(x)=2 \sum_{l=\widetilde{N}_{1}}^{\widetilde{N}_{2}} \widetilde{a}_{l} \widetilde{\varphi}(2 x-l)
$$

for every $x \in \mathbb{R}$. Let us set $a_{l}:=0$ for all $l \in \mathbb{Z} \backslash\left\{N_{1}, \ldots, N_{2}\right\}$ and $\widetilde{a}_{l}:=0$ for all $l \in \mathbb{Z} \backslash\left\{\widetilde{N}_{1}, \ldots, \widetilde{N}_{2}\right\}$. In this biorthogonal setting, Eq. (4) becomes

$$
\widetilde{m}_{0}(\xi) \overline{m_{0}(\xi)}+\widetilde{m}_{0}(\xi+\pi) \overline{m_{0}(\xi+\pi)}=1, \quad \forall \xi \in \mathbb{R},
$$

where

$$
m_{0}(\xi):=\sum_{l=N_{1}}^{N_{2}} a_{l} e^{-i l \xi} \text { and } \quad \widetilde{m}_{0}(\xi):=\sum_{l=\widetilde{N}_{1}}^{\widetilde{N}_{2}} \widetilde{a}_{l} e^{-i l \xi}, \quad \forall \xi \in \mathbb{R} .
$$

Let us note that (13) also becomes

$$
\psi(x)=2 \sum_{l \in \mathbb{Z}} b_{l} \varphi(2 x-l) \quad \text { and } \quad \widetilde{\psi}(x)=2 \sum_{l \in \mathbb{Z}} \widetilde{b}_{l} \widetilde{\varphi}(2 x-l)
$$

for every $x \in \mathbb{R}$, where

$$
b_{l}:=(-1)^{l-1} \widetilde{a}_{1-l} \quad \text { and } \quad \widetilde{b}_{l}:=(-1)^{l-1} a_{1-l}, \quad \forall l \in \mathbb{Z} .
$$

Moreover, the numbers $N_{2}-\widetilde{N}_{1}$ and $\widetilde{N}_{2}-N_{1}$ are positive and odd (see Ref. 10). Together with (201), this easily gives an equivalent of Lemma 3.2 for the sequences 
$\left(a_{l}\right)_{l \in \mathbb{Z}}$ and $\left(\widetilde{a}_{l}\right)_{l \in \mathbb{Z}}$. Consequently, the proof of Theorem 2.1 can be rewritten in a similar way, up to the equality (16) which becomes

$$
\begin{aligned}
&\left(\sum_{\widetilde{N}_{1} \leq 2 l+1 \leq \widetilde{N}_{2}} \widetilde{a}_{2 l+1} z^{2 l+1-\widetilde{N}_{1}}\right)\left(\sum_{\left\lfloor 2 x_{0}-N_{2}\right\rfloor \leq 2 q \leq\left\lfloor 2 x_{0}-N_{1}\right\rfloor} \varphi\left(2 x_{0}-2 q\right) z^{2 q-\left\lfloor 2 x_{0}-N_{2}\right\rfloor}\right) \\
&=\left(\sum_{\widetilde{N}_{1} \leq 2 l \leq \widetilde{N}_{2}} \widetilde{a}_{2 l} z^{2 l-\widetilde{N}_{1}}\right) \\
& \times\left(\sum_{\left\lfloor 2 x_{0}-N_{2}\right\rfloor \leq 2 q+1 \leq\left\lfloor 2 x_{0}-N_{1}\right\rfloor} \varphi\left(2 x_{0}-2 q-1\right) z^{2 q+1-\left\lfloor 2 x_{0}-N_{2}\right\rfloor}\right) .
\end{aligned}
$$

The last part of the proof can be easily adapted if $N_{2}-N_{1}=\widetilde{N}_{2}-\widetilde{N}_{1}$. In the case $N_{2}-N_{1}<\widetilde{N}_{2}-\widetilde{N}_{1}$, the generalization of Lemma 3.2 and the comparison of the degree of the polynomials appearing in (21) also lead to a contradiction. By interchanging the roles played by $\psi$ and $\widetilde{\psi}$ when $N_{2}-N_{1}>\widetilde{N}_{2}-\widetilde{N}_{1}$, one gets that for every $x_{0} \in \mathbb{R}$, there is $k_{x_{0}} \in \mathbb{Z}$ such that

$$
\psi\left(x_{0}-k_{x_{0}}\right) \neq 0 \quad \text { or } \quad \widetilde{\psi}\left(x_{0}-k_{x_{0}}\right) \neq 0 .
$$

\section{Acknowledgments}

The authors would like to thank the anonymous referee for her/his pertinent remarks which have led to improvements of the earlier version of their paper, and in particular for having pointed out the generalization to biorthogonal wavelets. The second author is supported by the Labex CEMPI (ANR-11-LABX-0007-01).

\section{References}

1. B. Arras, On a class of self-similar processes with stationary increments in higher order Wiener chaoses, Stochastic Process. Appl. 124(7) (2014) 2415-2441.

2. J.-M. Aubry, D. Maman and S. Seuret, Local behavior of traces of Besov functions: Prevalent results, J. Funct. Anal. 264(3) (2013) 631-660.

3. A. Ayache, Hausdorff dimension of the graph of the fractional Brownian sheet, Rev. Mat. Iberoamericana 20(2) (2004) 395-412.

4. A. Ayache, S. Jaffard and M. S. Taqqu, Wavelet construction of generalized multifractional processes, Rev. Mat. Iberoamericana 23(1) (2007) 327-370.

5. A. Ayache, F. Roueff and Y. Xiao, Linear fractional stable sheets: Wavelet expansion and sample path properties, Stochastic Process. Appl. 119(4) (2009) 1168-1197.

6. A. Ayache, N. R. Shieh and Y. Xiao, Multiparameter multifractional Brownian motion: Local nondeterminism and joint continuity of the local times, Ann. Inst. Henri Poincaré Probab. Stat. 47(4) (2011) 1029-1054.

7. A. Ayache and Y. Xiao, Asymptotic properties and Hausdorff dimensions of fractional Brownian sheets, J. Fourier Anal. Appl. 11(4) (2005) 407-439.

8. A. Benassi, S. Jaffard and D. Roux, Elliptic Gaussian random processes, Rev. Mat. Iberoamericana 13(1) (1997) 19-90. 
9. A. Cohen, Numerical Analysis of Wavelet Methods (Elsevier, Amsterdam, 2003).

10. A. Cohen, I. Daubechies and J.-C. Feauveau, Biorthogonal bases of compactly supported wavelets, Comm. Pure Appl. Math. 45 (1992) 485-560.

11. I. Daubechies, Ten Lectures on Wavelets, CBMS-NSF Regional Conference Series in Applied Mathematics (Society for Industrial and Applied Mathematics (SIAM), Philadelphia, 1992).

12. G. de Rham, Sur une courbe plane, J. Math. 35 (1956) 25-42.

13. G. Delauriers, J. Dubois and S. Dubuc, Multidimensional interative interpolation, Canad. J. Math. 43 (1991) 297-312.

14. C. Esser and S. Jaffard, Divergence of wavelet series: A multifractal analysis, submitted for publication (2016).

15. E. Hernández and G. Weiss, A First Course on Wavelets (CRC Press, New York, 1996).

16. Y. Kozachenko, A. Olenko and O. Polosmak, Uniform convergence of compactly supported wavelet expansions of Gaussian random processes, Comm. Statist. Theory Methods 43(10-12) (2014) 2549-2562.

17. Y. Kozachenko, A. Olenko and O. Polosmak, Convergence in $L_{p}([0, T])$ of wavelet expansions of $\phi$-sub-Gaussian random processes, Methodol. Comput. Appl. Probab. 17(1) (2015) 139-153.

18. W. M. Lawton, Tight frames of compactly supported affine wavelets, J. Math. Phys. 31(8) (1990) 1898-1901.

19. P. Lemarié, On the existence of compactly supported dual wavelets, Appl. Comput. Harmon. Anal. 4 (1997) 117-118.

20. P. Lemarié and G. Malgouyres, Support des fonctions de base dans une analyse multirésolution, C. R. Acad. Sci. Paris Sér. I Math. 313(6) (1991) 377-380.

21. P. G. Lemarié and Y. Meyer, Ondelettes et bases hilbertiennes, Rev. Math. Iberoamericana 1 (1986) 1-18.

22. Y. Meyer, Ondelettes et opérateurs (Hermann, 1990).

23. Y. Meyer, F. Sellan and M. S. Taqqu, Wavelets, generalized white noise and fractional integration: The synthesis of fractional Brownian motion, J. Fourier Anal. Appl. 5(5) (1999) 465-494.

24. C. A. Michelli and H. Prautzsch, Uniform refinement of curves, Linear Algebra Appl. 114 (1989) 841-870.

25. F. Roueff, Dimension de Hausdorff du graphe d'une fonction continue: une étude analytique et statistique, $\mathrm{PhD}$ thesis, École Nationale Supérieure des Télécommunications (2000).

26. F. Roueff, Almost sure Hausdorff dimension of graphs of random wavelet series, J. Fourier Anal. Appl. 9 (2003) 237-260. 